\title{
APPROXIMATION OF INTEGRATION MAPS OF VECTOR MEASURES AND LIMIT REPRESENTATIONS OF BANACH FUNCTION SPACES
}

\author{
EDUARDO JIMÉNEZ FERNÁNDEZ, ENRIQUE A. SÁNCHEZ PÉREZ, \\ AND DIRK WERNER
}

\begin{abstract}
We study when the integration maps of vector measures can be computed as pointwise limits of their finite rank Radon-Nikodým derivatives. We will show that this can sometimes be done, but there are also principal cases in which this cannot be done. The positive cases are obtained using the circle of ideas of the approximation property for Banach spaces. The negative ones are given by means of an adequate use of the Daugavet property. As an application, we analyse when the norm in a space of integrable functions $L^{1}(m)$ can be computed as a limit of the norms of the spaces of integrable functions with respect to the Radon-Nikodým derivatives of $m$.
\end{abstract}

\section{INTRODUCTION}

Let $X$ be a Banach space and $m$ be an $X$-valued countably additive vector measure. Consider the space $L^{1}(m)$ of (scalar) integrable functions with respect to $m$. In this paper we are interested in the analysis of the (pointwise) approximation by means of finite rank operators of the associated integration map $I_{m}: L^{1}(m) \rightarrow$ $X$. Actually, we will choose nets of finite rank operators of a special class, which are the finite rank Radon-Nikodým derivatives of $m$.

We will show that this can be done in the case that we consider weakly compact operators having values in a space that has the approximation property. This allows us to prove a positive result for a big class of usual examples, as for instance when $X$ is a reflexive space. However, there are cases - and some of them can even be considered as canonical - in which these results cannot be applied. Martingale type procedures also allow us to find approximations of integration maps by finite rank operators for some of these situations, for example the identity map (considered as an integration operator) when $m$ is a (scalar) probability measure without atoms, and so the space $L^{1}(m)$ is a classical Lebesgue $L^{1}$-space. However, we show also that the unconditional pointwise approximation by series of finite rank operators has some constraints, and cannot be expected in general. For doing this, we will use the circle of ideas of the Daugavet property. A Banach space $X$ is said to have the Daugavet property if every rank one operator $T: X \rightarrow X$ satisfies the Daugavet

2010 Mathematics Subject Classification. Primary 46G05, 28B05; Secondary 46E30, 47B07, 47B38.

Key words and phrases. Vector measures, integration map, Daugavet property.

E. Jiménez Fernández was supported by Junta de Andalucía and FEDER grant and P09FQM-4911 (Spain) and by Ministerio de Economía, Industria y Competitividad (Spain) (project MTM2012-36740-C02-02). E.A. Sánchez Pérez was supported by Ministerio de Economía, Industria y Competitividad (Spain) (project MTM2016-77054-C2-1-P). 
equation, that is $\|\operatorname{Id}+T\|=1+\|T\|$. In recent years, great efforts have been made for studying the Daugavet property for Banach spaces and Banach lattices, and its natural extension to other operators different from the identity map, which are the so-called Daugavet centers. The reader can find also information about the Daugavet equation for general maps [5] and the references therein.

The reason why this property is relevant for us is that the Daugavet equation as an estimate of the norm of the sum of a pair of operators may be used for establishing clear criteria of when a particular map cannot be approximated pointwise unconditionally by series of finite rank operators; see for example Theorem 2.9 in [4]. Therefore, for establishing some limits to the approximation by finite rank maps, we are interested in analysing when the integration operator $I_{m}: L^{1}(m) \rightarrow X$ satisfies the Daugavet equation.

\section{Preliminaries}

The notation that we use is standard. If $X$ is a Banach space, we write $B_{X}$ for its closed unit ball, and $X^{*}$ for its dual space. Let $(\Omega, \Sigma, \mu)$ be a positive finite measure space, and write $L^{0}(\mu)$ for the space of all measurable real functions on $\Omega$ (functions which are equal $\mu$-a.e. are identified). We say that a Banach space $X(\mu)$ of functions in $L^{0}(\mu)$ is a Banach function space with respect to $\mu$ if $\chi_{\Omega} \in X(\mu)$, and for each pair of measurable functions $f, g$, if $|f| \leq|g|$ with $g \in X(\mu)$, then $f \in X(\mu)$ and $\|f\| \leq\|g\|$. We will write $X$ instead of $X(\mu)$ if the measure $\mu$ is fixed in the context. We say that $X(\mu)$ is $\sigma$-order continuous if for every sequence of functions $f_{n} \in X(\mu)$ with $f_{n} \downarrow 0$ it follows that $\left\|f_{n}\right\|_{X(\mu)} \rightarrow 0$. We will also use the following (non standard) notation. If $\mu$ and $\nu$ are measures on the same measurable space and $\nu$ is absolutely continuous with respect to $\mu$, we will write $X(\mu) \Subset Y(\nu)$ if the operator that maps the class of $\mu$-a.e. equal functions of $f$ to the class of $\nu$-a.e. equal functions of the same $f$ is continuous.

Throughout the paper $m: \Sigma \rightarrow X$ will be a countably additive vector measure, where $E$ is a Banach space; the reader can find all the information that is needed - and shortly explained below - on vector measures and integration in [6, 14]. For each element $x^{*} \in X^{*}$ the formula $\left\langle m, x^{*}\right\rangle(A):=\left\langle m(A), x^{*}\right\rangle, A \in \Sigma$, defines a (countably additive) scalar measure. We write $\left|\left\langle m, x^{*}\right\rangle\right|$ for its variation. The function $\|m\|$ given on a set $A \in \Sigma$ by

$$
\|m\|(A)=\sup \left\{\left|\left\langle m, x^{\prime}\right\rangle\right|(A): x^{*} \in X^{*},\left\|x^{*}\right\| \leq 1\right\}
$$

is called the semivariation of $m$. It is equivalent to the variation if $m$ is a scalar measure. A (vector or scalar valued) measure $m$ is absolutely continuous with respect to $\mu$ if $\mu(A)=0$ implies $\|m\|(A)=0$; in this case we write $m \ll \mu$. It is well known that there is always a (non-unique) measure of the form $\left|\left\langle m, x^{*}\right\rangle\right|$ such that $m$ is absolutely continuous with respect to it; we will call such a measure a Rybakov measure for $m$. Let $\mu$ be a finite measure; we say that a Banach space valued vector measure $m: \Sigma \rightarrow X$ is equivalent to $\mu$ if for all $A \in \Sigma, \mu(A)=0$ if and only if $\|m\|(A)=0$.

The space $L^{1}(m)$ of integrable functions with respect to $m$ is a Banach function space over any Rybakov measure; throughout the paper, we will fix one of them, which will usually be denoted by $\mu$. The elements of this space are (classes of $\mu$-a.e. measurable) functions $f$ that are integrable with respect to each scalar measure $\left\langle m, x^{*}\right\rangle$, and for every $A \in \Sigma$ there is an element $\int_{A} f d m \in X$ such that 
$\left\langle\int_{A} f d m, x^{*}\right\rangle=\int_{A} f d\left\langle m, x^{*}\right\rangle$ for every $x^{*} \in X^{*}$; the space $L^{1}(m)$ of $m$-a.e. equal $m$-integrable functions is an order continuous Banach lattice endowed with the norm

$$
\|f\|_{L^{1}(m)}:=\sup _{x^{*} \in B_{X^{*}}} \int|f| d\left|\left\langle m, x^{*}\right\rangle\right|, \quad f \in L^{1}(m),
$$

and the $m$-a.e. order. The associated integration operator $I_{m}: L^{1}(m) \rightarrow X$ is defined by $I_{m}(f):=\int_{\Omega} f d m$, where $f \in L^{1}(m)$. It is linear and continuous and $\left\|I_{m}\right\|=1$.

A continuous operator $T: X \rightarrow X$ on a Banach space is said to satisfy the Daugavet equation if the following formula holds,

$$
\|\operatorname{Id}+T\|=1+\|T\| .
$$

A Banach space $X$ is said to satisfy the Daugavet property if $\|\mathrm{Id}+T\|=1+\|T\|$ is satisfied for every rank one operator. It is well known that, if this happens, then the same equation holds for each weakly compact or merely Radon-Nikodým operator 9. Recall that a subset $A$ of a Banach space is said to have the RadonNikodým property if every closed convex subset $B \subseteq A$ is the closed convex hull of its denting points; an operator $T$ is said to be a Radon-Nikodým operator if the closure of $T\left(B_{X}\right)$ has the Radon-Nikodým property. Weakly compact operators belong to this class.

The reader can find a review on the classical results on the Daugavet property in 16]; for the case of Banach lattices of functions, which is particularly important for this paper, see [1, 2] and the references therein. The following generalization of the notion of Daugavet property will be used. Following [4, Definition 1.2], we say that a continuous linear operator $G: X \rightarrow Y$ between Banach spaces is a Daugavet center if $\|G+T\|=\|G\|+\|T\|$ is fulfilled for every rank-1 operator $T: X \rightarrow Y$. For this notion and the main properties which are necessary for this paper, see also [3, 4.

\section{ApProximation OF WEAKLY COMPACT INTEGRATION OPERATORS OF A VECTOR MEASURE BY ITS FINITE RANK RADON-NIKODÝM DERIVATIVES}

The approximation of weakly compact operators by means of finite rank operators is closely related to the approximation property of the Banach space $X$ where the operators are defined, or to this property for its dual space $X^{*}$. A series of papers (e.g., [12]) recently published have shown that $X^{*}$ has the approximation property if and only if for every Banach space $Z$ and every weakly compact operator $T: Z \rightarrow X$ there is a net of finite rank operators $T_{\alpha}: Z \rightarrow X$ of norm $\left\|T_{\alpha}\right\| \leq\|T\|$ converging to $T$ pointwise. The problem goes back to the memoir of Grothendieck [7. p. 184], and these developments have provided some useful tools and solutions to some long-standing questions that will provide the key for our arguments. The question is: When and how can an integration map of a vector measure be approximated by nets of finite rank operators? As the reader will see, the result will be used to study when, given a vector measure $m$, the norm of each element in the corresponding space of integrable functions can be approximated as a limit of a net of the natural finite components of $I_{m}$, which we call finite rank Radon-Nikodým derivative operators of $m$. 
3.1. Integration operators and Radon-Nikodým derivatives of vector measures. Let us start our analysis by establishing several results regarding the set of Radon-Nikodým derivatives of the scalarizations of a given vector measure. Let $m: \Sigma \rightarrow E$ be a (countably additive) vector measure.

Lemma 3.1. Consider a vector measure $m: \Sigma \rightarrow X$ and a fixed finite measure $\mu$ equivalent to $m$ representing the duality (that is for $f \in L^{1}(m)$ and $g \in\left(L^{1}(m)\right)^{\prime}$, the Köthe dual, $\langle f, g\rangle:=\int f g d \mu$; it may be a Rybakov measure). Then for every $x^{*} \in X^{*}$,

$$
I_{m}^{*}\left(x^{*}\right)=\varphi_{x^{*}}^{m}:=\frac{d\left\langle m, x^{*}\right\rangle}{d \mu} \in\left(L^{1}(m)\right)^{\prime},
$$

where $\frac{d\left\langle m, x^{*}\right\rangle}{d \mu}$ represents the Radon-Nikodym derivative of the measure $A \mapsto\left\langle m(A), x^{*}\right\rangle$ with respect to $\mu$. Moreover, $\left\|\varphi_{x^{*}}^{m}\right\|_{\left(L^{1}(m)\right)^{\prime}} \leq\left\|x^{*}\right\|_{X^{*}}$ for all $x^{*} \in X^{*}$.

Proof. Take a function $f \in L^{1}(m)$ and $x^{*} \in X^{*}$. Then, since $f$ is $m$-integrable and $\left\langle m, x^{*}\right\rangle \ll \mu$, we have

$$
\left\langle I_{m}(f), x^{*}\right\rangle=\int f d\left\langle m, x^{*}\right\rangle=\int f \frac{d\left\langle m, x^{*}\right\rangle}{d \mu} d \mu=\int f \varphi_{x^{*}}^{m} d \mu .
$$

Since this is well-defined for each $f \in L^{1}(m)$, we have that $\varphi_{x^{*}}^{m} \in\left(L^{1}(m)\right)^{\prime}$; more specifically,

$$
\left|\int f \frac{d\left\langle m, x^{*}\right\rangle}{d \mu} d \mu\right| \leq\|f\|_{L^{1}(m)} \cdot\left\|\frac{d\left\langle m, x^{*}\right\rangle}{d \mu}\right\|_{\left(L^{1}(m)\right)^{\prime}} .
$$

Moreover,

$$
\begin{aligned}
\left\|\varphi_{x^{*}}^{m}\right\|_{\left(L^{1}(m)\right)^{\prime}} & =\sup _{f \in B_{L^{1}(m)}}\left|\int f \frac{d\left\langle m, x^{*}\right\rangle}{d \mu} d \mu\right|=\sup _{f \in B_{L^{1}(m)}}\left|\left\langle\int f d m, x^{*}\right\rangle\right| \\
& \leq \sup _{f \in B_{L^{1}(m)}}\left\|\int f d m\right\| \cdot\left\|x^{*}\right\|_{X^{*}} \leq\left\|x^{*}\right\|_{X^{*}}
\end{aligned}
$$

Note, however, that the map $x^{*} \mapsto \varphi_{x^{*}}^{m}$ need not be injective in general.

3.2. Pointwise convergence of nets of norms to the norm in $L^{1}(m)$. In this section we prove the main general result concerning convergence of nets of norms of spaces $L^{1}\left(m_{\eta}\right)$ to $\|\cdot\|_{L^{1}(m)}$.

Fix a finite measure $\mu$. Let $\left\{X_{\tau}\left(\mu_{\tau}\right): \tau \in \Lambda\right\}$ be a net of spaces, where all the measures $\mu_{\tau}$ are absolutely continuous with respect to $\mu$. We will study when a Banach function space $X(\mu)$ such that $X(\mu) \subseteq \bigcap_{\tau \in \Lambda} X_{\tau}\left(\mu_{\tau}\right)$ can be computed as a pointwise limit of the norms as $\lim _{\tau}\|f\|_{X_{\tau}\left(\mu_{\tau}\right)}=\|f\|_{X(\mu)}$ for all $f \in X(\mu)$.

In what follows we are interested in analysing the properties under which we have that a net of vector measures $\left\{m_{\tau}\right\}$ gives that the norm in $L^{1}(m)$ is a pointwise limit of $\left\{L^{1}\left(m_{\tau}\right)\right\}$. We need to introduce some new elements.

Let $A \in \Sigma$. We define $h_{A}$ as the function in $L^{\infty}(\mu)$ given by $h_{A}=\chi_{A}-\chi_{A^{c}}$; that is, the definition makes sense $\mu$-a.e. We will denote by $\mathcal{H}$ all the (classes of) functions defined in this way.

Lemma 3.2. Let $m: \Sigma \rightarrow X$ be a countably additive vector measure. For all functions $f \in L^{1}(m)$, we have

$$
\|f\|_{L^{1}(m)}=\sup _{A \in \Sigma}\left\|\int f h_{A} d m\right\|_{X} .
$$


Proof. This is a consequence of a direct calculation; we write $A\left(x^{*}\right)$ for the measurable set, depending on $x^{*}$, where the Radon-Nikodým derivative of $\left\langle m, x^{*}\right\rangle$ with respect to $\left|\left\langle m, x^{*}\right\rangle\right|$ equals 1 . Then

$$
\begin{aligned}
\|f\|_{L^{1}(m)}= & \sup _{x^{*} \in B_{X^{*}}} \int|f| d\left|\left\langle m, x^{*}\right\rangle\right| \\
= & \sup _{x^{*} \in B_{X^{*}}} \int f\left(\chi_{\{\operatorname{sign}(f)>0\}}-\chi_{\{\operatorname{sign}(f) \leq 0\}}\right)\left(\chi_{A\left(x^{*}\right)}-\chi_{A\left(x^{*}\right)^{c}}\right) d\left\langle m, x^{*}\right\rangle \\
= & \sup _{x^{*} \in B_{X^{*}}}\left[\int f\left(\chi_{\left.\left.\{\operatorname{sign}(f)>0\} \cap A\left(x^{*}\right)\right\} \cup\{\operatorname{sign}(f) \leq 0\} \cap A\left(x^{*}\right)^{c}\right\}}\right) d\left\langle m, x^{*}\right\rangle\right. \\
& \left.-\int f\left(\chi_{\left.\left.\{\operatorname{sign}(f)>0\} \cap A\left(x^{*}\right)^{c}\right\} \cup\{\operatorname{sign}(f) \leq 0\} \cap A\left(x^{*}\right)\right\}}\right) d\left\langle m, x^{*}\right\rangle\right] .
\end{aligned}
$$

Since the sets appearing in the characteristic functions in the expression above are complementary and using the fact that the function is integrable with respect to $m$, we have that this expression is

$$
\leq \sup _{x^{*} \in B_{X^{*}}, A \in \Sigma}\left\langle\int f h_{A} d m, x^{*}\right\rangle=\sup _{A \in \Sigma}\left\|\int f h_{A} d m\right\|_{X} .
$$

The converse inequality is a direct consequence of the fact that $|f|=\left|f h_{A}\right|$ for all $A \in \Sigma,\|\cdot\|_{L^{1}(m)}$ is a lattice norm, and the straightforward inequality

$$
\left\|\int f h_{A} d m\right\|_{X} \leq\left\|f h_{A}\right\|_{L^{1}(m)}=\|f\|_{L^{1}(m)} .
$$

Lemma 3.3. Let $m, m_{1}: \Sigma \rightarrow X$ be a couple of vector measures and consider a function $f \in L^{1}(m) \cap L^{1}\left(m_{1}\right)$. Then

$$
\left|\|f\|_{L^{1}(m)}-\|f\|_{L^{1}\left(m_{1}\right)}\right| \leq \sup _{A \in \Sigma}\left\|\int f h_{A} d m-\int f h_{A} d m_{1}\right\|_{X} .
$$

Proof. Consider the linear space $V M$ of all countably additive vector measures from $\Sigma$ to $X$. Fix a simple function $f$. The function $\phi_{f}: \mathcal{H} \times V M \rightarrow X$ given by the formula

$$
\phi_{f}\left(h_{A}, m\right):=\int f h_{A} d m \in X
$$

is well-defined. Note that

$$
m \mapsto \sup _{A \in \Sigma}\left\|\int f h_{A} d m\right\|_{X}
$$

is a seminorm on $V M$. Consequently, if $m, m_{1} \in V M$ we have that

$$
\left|\sup _{A \in \Sigma}\left\|\int f h_{A} d m\right\|_{X}-\sup _{A \in \Sigma}\left\|\int f h_{A} d m_{1}\right\|_{X}\right| \leq \sup _{A \in \Sigma}\left\|\int f h_{A} d\left(m-m_{1}\right)\right\|_{X}
$$

for all simple functions. Thus, as a consequence of Lemma 3.2, we get

$$
\left|\|f\|_{L^{1}(m)}-\|f\|_{L^{1}\left(m_{1}\right)}\right| \leq \sup _{A \in \Sigma}\left\|\int f h_{A} d m-\int f h_{A} d m_{1}\right\|_{X}
$$

for all simple functions. Since simple functions are dense in both spaces for the norm, we get the result for all the functions in $L^{1}(m) \cap L^{1}\left(m_{1}\right)$. 
The next result provides our first general approximation theorem, which can be applied to any net of Banach space valued vector measures. Notice that no requirements on $X$ are imposed. Later on in this section we will show that this situation can always be given - that is, there always exists such a net for any vector measure - whenever $X$ has the approximation property and the integration map is weakly compact. Recall from Lemma 3.1 the definition of the Radon-Nikodým derivatives $\varphi_{x^{*}}^{m}$ of the measure $m$.

Theorem 3.4. Let $m, m_{\eta}: \Sigma \rightarrow X$ be countably additive vector measures all of them absolutely continuous with respect to $\mu$, with $\eta \in \Lambda$, a directed set such that $L^{1}(m) \subseteq \bigcap_{\eta \in \Lambda} L^{1}\left(m_{\eta}\right)$ with norm one for the inclusion in each space $L^{1}\left(m_{\eta}\right)$. Assume that we have one of the following requirements for the net $\left\{m_{\eta}: \eta \in \Lambda\right\}$ :

(i) For each $f \in L^{1}(m)$,

$$
\lim _{\eta} \sup _{A \in \Sigma}\left\|\int f h_{A} d m-\int f h_{A} d m_{\eta}\right\|=0 .
$$

(ii) For each $x^{*} \in X^{*}$,

$$
\lim _{\eta} \varphi_{x^{*}}^{m_{\eta}}=\varphi_{x^{*}}^{m}
$$

in the weak ${ }^{*}$ topology.

Then for each $f \in L^{1}(m)$,

$$
\lim _{\eta \in \Lambda}\|f\|_{L^{1}\left(m_{\eta}\right)}=\|f\|_{L^{1}(m)} .
$$

Proof. Assume first that (i) holds. Then the result is a consequence of Lemma 3.3 . Using the inequality given there and taking into account that each $m$-integrable function is $m_{\eta}$-integrable, we get that for a fixed $f$ in $L^{1}(m)$,

$$
\|\| f\left\|_{L^{1}(m)}-\right\| f\left\|_{L^{1}\left(m_{\eta}\right)} \mid \leq \sup _{A \in \Sigma}\right\| \int f h_{A} d m-\int f h_{A} d m_{\eta} \|_{X} \rightarrow_{\eta} 0 .
$$

Suppose now that (ii) holds. Fix $f \in L^{1}(m)$ and $\varepsilon>0$. Taking into account Lemma 3.1 we know that there are $x^{*} \in B_{X^{*}}$ and $A \in \Sigma$ such that

$$
\int f h_{A} d\left\langle m, x^{*}\right\rangle \geq\|f\|_{L^{1}(m)}-\varepsilon
$$

Then, using the pointwise limit condition, we find

$$
\lim _{\eta} \varphi_{x^{*}}^{m_{\eta}}\left(f h_{A}\right)=\varphi_{x^{*}}^{m}\left(f h_{A}\right)=\int f h_{A} d\left\langle m, x^{*}\right\rangle \geq\|f\|_{L^{1}(m)}-\varepsilon .
$$

Since for all $\eta$ we have that $\int f h_{A} d\left\langle m_{\eta}, x^{*}\right\rangle \leq\|f\|_{L^{1}\left(m_{\eta}\right)}$, we obtain that there is $\eta_{0} \in \Lambda$ such that for all $\eta \geq \eta_{0}$,

$$
\|f\|_{L^{1}\left(m_{\eta}\right)}+\varepsilon \geq\|f\|_{L^{1}(m)}-\varepsilon .
$$

On the other hand, $\|f\|_{L^{1}(m)} \geq\|f\|_{L^{1}\left(m_{\eta}\right)}$, and since this happens for each $\varepsilon>0$ we obtain

$$
\|f\|_{L^{1}(m)}=\lim _{\eta}\|f\|_{L^{1}\left(m_{\eta}\right)} .
$$


3.3. Approximation of weakly compact integration maps. The arguments in the previous sections lead to the main result of this section. We want to know if it is possible to approximate the integration map by means of finite rank operators. In fact, we want to know how well an integration map $I_{m}: L^{1}(m) \rightarrow X$ can be approximated using the natural associated finite rank operators, which are the finite rank Radon-Nikodým derivatives of $m$ to be defined later on. Let us start with an easy case - vector measures with values in a Banach space with a Schauder basis in order to show the arguments that we use.

Example 3.5. Let us consider now the approximation of integration operators of vector measures with values in a Banach space with a Schauder basis. Let us show that in this case, nothing is required for obtaining a pointwise approximation of the norm of the space $L^{1}(m)$. Let us explain this case, which allows a specific treatment using some ad hoc constructed tools.

Let $X=\ell$ be a Banach space with a normalized monotone Schauder basis $\left\{e_{i}\right\}_{i=1}^{\infty}$; hence we assume that the basic constant is 1 . We can define the sequence of biorthogonal functionals $\left\{e_{i}^{*}\right\}_{i=1}^{\infty}$ in $\ell^{*}$ as usual: for each $i, j \in \mathbb{N}, e_{i}^{*}\left(e_{j}\right)=\delta_{i, j}$, Kronecker's delta of $i$ and $j$. For a fixed natural number $n$, write $P_{n}$ for the basis projection on the $n$-dimensional space generated by the first $n$ vectors, that is

$$
P_{n}(x):=\sum_{i=1}^{n}\left\langle x, e_{i}^{*}\right\rangle e_{i} \in \ell, \quad x \in \ell
$$

we have $\left\|P_{n}\right\| \leq 1$ by assumption. Consider a countably additive vector measure $m: \Sigma \rightarrow \ell$ and construct the finite dimensional components $m_{n}$ of the measure by

$$
m_{n}(A):=\left(P_{n} \circ m\right)(A)=\sum_{i=1}^{n}\left\langle m(A), e_{i}^{*}\right\rangle e_{i} \in \ell, \quad A \in \Sigma, n \in \mathbb{N} .
$$

This clearly provides a sequence of countably additive vector measures. Take a function $f \in L^{1}(m)$. Then we have that for each fixed $A \in \Sigma$

$$
\left\|\int f h_{A} d m\right\|_{\ell}=\lim _{n}\left\|P_{n}\left(\int f h_{A} d m\right)\right\|_{\ell}=\lim _{n}\left\|\int f h_{A} d m_{n}\right\|_{\ell} .
$$

Thus,

$$
\|f\|_{L^{1}(m)}=\sup _{A \in \Sigma}\left\|\int f h_{A} d m\right\|_{\ell}=\sup _{A \in \Sigma}\left(\lim _{n}\left\|\int f h_{A} d m_{n}\right\|_{\ell}\right) .
$$

On the other hand, using that $\left\|P_{n}\right\| \leq 1$, we obtain

$$
\sup _{A \in \Sigma}\left\|\int f h_{A} d m\right\|_{\ell} \geq \sup _{A \in \Sigma}\left\|P_{n}\left(\int f h_{A} d m\right)\right\|_{\ell}=\sup _{A \in \Sigma}\left\|\int f h_{A} d m_{n}\right\|_{\ell}
$$

for every $n \in \mathbb{N}$, and so

$$
\left.\|f\|_{L^{1}(m)}=\sup _{A \in \Sigma}\left(\lim _{n}\left\|\int f h_{A} d m_{n}\right\|_{\ell}\right) \leq \lim _{n}\left(\sup _{A \in \Sigma} \| \int f h_{A} d m_{n}\right) \|_{\ell}\right) \leq\|f\|_{L^{1}(m)} .
$$

This proves the result.

The same arguments prove the following general result.

Proposition 3.6. Let $m$ be a vector measure. Consider a net $\left(P_{\eta}\right)$ of operators $P_{\eta}: X \rightarrow X$ that converges pointwise to the identity map in $X$, and such that for 
all $\eta,\left\|P_{\eta}\right\| \leq 1$. Consider the vector measures $m_{\eta}:=P_{\eta} \circ m$. Then $L^{1}(m) \Subset L^{1}\left(m_{\eta}\right)$ for all $\eta$ and for all $f \in L^{1}(m)$,

$$
\|f\|_{L^{1}(m)}=\lim _{\eta}\|f\|_{L^{1}\left(m_{\eta}\right)} .
$$

The natural finite rank maps in the setting of the vector measures are the ones that can be written as finite sums of products of vectors on $X$ by Radon-Nikodým derivatives of the scalarizations of the vector measure, that is, finite rank operators as

$$
R_{m}(\cdot)=\sum_{i=1}^{n} \varphi_{x_{i}^{*}}^{m}(\cdot) x_{i}=\sum_{i=1}^{n} \frac{d\left\langle m, x_{i}^{*}\right\rangle}{d \mu}(\cdot) x_{i} .
$$

We will call this kind of operator a finite rank Radon-Nikodým derivative operator of $m$.

Theorem 3.7. Let $m: \Sigma \rightarrow X$ a countably additive vector measure such that $I_{m}$ is weakly compact, where $X$ has the approximation property. Then there is a net of finite rank Radon-Nikodym derivative operators $\left(R_{m}^{\alpha}\right)_{\alpha}$ of norm $\leq 1$ that converges to $I_{m}$ in the strong operator topology. That is, for every $f \in L^{1}(m)$

$$
\lim _{\alpha} R_{m}^{\alpha}(f)=\int f d m=I_{m}(f) .
$$

Proof. We use Theorem 1.2 in [1], in which the famous Davis-Figiel-JohnsonPełczýnski factorization technique for weakly compact operators is used. Suppose that $X$ has the approximation property. Assume that $\left\|I_{m}\right\|=1$. Lemma 1.1 in 11. gives that there is a reflexive Banach space $X_{K}$ that is embedded in $X$ with inclusion map $J_{K}: X_{K} \rightarrow X$ in such a way that $I_{m}\left(B_{L^{1}(m)}\right) \subseteq B_{X_{K}}$. That is, there is a factorization of $T$ as $J_{K} \circ I_{m}^{0}$ through $X_{K}$, where $I_{m}^{0}: L^{1}(m) \rightarrow X_{K}$ is the integration map when restricted in the range to $X_{K}$, and $\left\|J_{K}\right\| \leq 1$. In the proof of Theorem 1.2 of 11 it can be seen that there exists a net $\left(A_{\alpha}\right)$ of finite rank operators from $X_{K}$ to $X$ such that $\sup _{\alpha}\left\|A_{\alpha}\right\| \leq 1$, with $A_{\alpha}\left(I_{m}^{0}(f)\right) \rightarrow J_{K}\left(I_{m}^{0}(f)\right)$ for all $f \in L^{1}(m)$. Define $R_{m}^{\alpha}(f):=A_{\alpha} \circ I_{m}^{0}(f)$. Each $A_{\alpha}$ has the form $A_{\alpha}=\sum_{i=1}^{n} z_{i}^{*} \otimes x_{i}$. Since $X_{K}$ is reflexive and $J_{K}$ is injective (cf. Lemma 1.1 in [11) we have that $J_{K}^{*}$ has dense range. Hence we may assume that $z_{i}^{*}=J_{K}^{*} x_{i}^{*}$, and we still have $\sup _{\alpha}\left\|A_{\alpha}\right\| \leq 1$ and $A_{\alpha}\left(I_{m}^{0}(f)\right) \rightarrow J_{K}\left(I_{m}^{0}(f)\right)$ for all $f \in L^{1}(m)$. Now we see that

$$
A_{\alpha}\left(I_{m}^{0}(f)\right)=\sum_{i=1}^{n} x_{i}\left\langle z_{i}^{*}, \int f d m\right\rangle=\sum_{i=1}^{n} x_{i}\left\langle x_{i}^{*}, \int f d m\right\rangle=\sum_{i=1}^{n}\left\langle f, \frac{d\left\langle m, x_{i}^{*}\right\rangle}{d \mu}\right\rangle x_{i}
$$

in the first instance, $\int f d m$ is taken in $X_{K}$ and in the second in $X$.

This gives the result.

The following is the main positive result regarding approximation of the norm of a space $L^{1}(m)$ by means of the finite dimensional components of the integration map $I_{m}$. Recall that, for an operator $T: L^{1}(m) \rightarrow X$, the associated vector measure is given by $m_{T}(A)=T\left(\chi_{A}\right), A \in \Sigma$.

Corollary 3.8. Let $m: \Sigma \rightarrow X$ be a countably additive vector measure such that $I_{m}$ is weakly compact, where $X$ has the approximation property. Then there is a net of finite rank Radon-Nikodým derivative operators $\left(R_{m}^{\alpha}\right)_{\alpha}$ such that

$$
\lim _{\alpha}\|f\|_{L^{1}\left(m_{\alpha}\right)}=\|f\|_{L^{1}(m)}, \quad f \in L^{1}(m),
$$

where $m_{\alpha}$ is the vector measure associated to $R_{m}^{\alpha}$. 
Proof. Consider the net of finite rank operators $\left(R_{m}^{\alpha}\right)_{\alpha}$ of norm $\leq 1$ that Theorem 3.7 provides. Note that all the measures $m_{\alpha}$ are countably additive, since $R_{m}^{\alpha}$ are finite rank operators and $L^{1}(m)$ is order continuous. Take a function $f \in L^{1}(m)$ and $\varepsilon>0$. Then there is $A \in \Sigma$ such that

$\|f\|_{L^{1}(m)}-\varepsilon<\left\|\int f h_{A} d m\right\|_{X}=\lim _{\alpha}\left\|\int f h_{A} d m_{\alpha}\right\|_{X} \leq \sup _{A \in \Sigma}\left(\lim _{\alpha}\left\|\int f h_{A} d m_{\alpha}\right\|_{X}\right)$.

Now, since $\left\|R_{\alpha}^{m}\right\| \leq 1$, we obtain

$$
\sup _{A \in \Sigma}\left\|\int f h_{A} d m\right\|_{X} \geq \sup _{A \in \Sigma}\left\|R_{\alpha}^{m}\left(f h_{A}\right)\right\|_{X}=\sup _{A \in \Sigma}\left\|\int f h_{A} d m_{\alpha}\right\|_{X}=\|f\|_{L^{1}\left(m_{\alpha}\right)}
$$

for every $\alpha$, which gives

$$
\begin{aligned}
\|f\|_{L^{1}(m)}-\varepsilon & <\sup _{A \in \Sigma}\left(\lim _{\alpha}\left\|\int f h_{A} d m_{\alpha}\right\|_{X}\right) \\
& \leq \lim _{\alpha}\left(\sup _{A \in \Sigma}\left\|\int f h_{A} d m_{\alpha}\right\|_{X}\right) \\
& \leq \lim _{\alpha}\|f\|_{L^{1}\left(m_{\alpha}\right)} \leq\|f\|_{L^{1}(m)} .
\end{aligned}
$$

This gives the proof.

This clarifies the situation in a great class of Banach space valued vector measures. For example, if $X$ is reflexive we obtain the result directly: each integration map can be approximated by a net of finite rank operators - that in fact defines a net of vector measures $\left(m_{\alpha}\right)_{\alpha}$-, and its norm can be computed as the limit of the norms in the associated $L^{1}\left(m_{\eta}\right)$-spaces.

3.4. Approximation of the integration map by martingale type constructions. In the previous section we have shown that weakly compact integration operators in spaces with the approximation property allow approximations by finite rank Radon-Nikodým derivative operators. However, this result still excludes the canonical example of integration map: the identity map in $L^{1}[0,1]$. The method of approximation that can be used in this case is based on martingale type constructions. Consider a vector measure $m: \Sigma \rightarrow X$ and the space of integrable functions $L^{1}(m)$. Let $\mathbb{P}:=\left\{\mathcal{P}_{\eta}: \eta \in \Lambda\right\}$ be the net of (classes of $\mu$-a.e. equal) finite measurable partitions of $\Omega$ endowed with the usual inclusion order. Consider the net of vector measures

$$
m_{\eta}(A):=\sum_{B \in \mathcal{P}_{\eta}} \frac{\mu(A \cap B)}{\mu(B)} m(B) \in X,
$$

where $\mathcal{P}_{\eta} \in \mathbb{P}$.

Consider the corresponding integration map $I_{m_{\eta}}: L^{1}\left(m_{\eta}\right) \rightarrow X$. It is easy to see that this is given by the formula

$$
I_{m_{\eta}}(f):=\sum_{B \in \mathcal{P}_{\eta}} \frac{\int_{B} f d \mu}{\mu(B)} m(B) \in X, \quad f \in L^{1}\left(m_{\eta}\right) .
$$

A direct computation shows also that $L^{1}(m) \Subset L^{1}\left(m_{\eta}\right)$, that is, $I_{m_{\eta}}$ is well-defined for all functions in $L^{1}(m)$. The question then is: When can the integration map 
$I_{m}$ be approximated pointwise by the family $\left\{I_{m_{\eta}}: \eta \in \Lambda\right\}$ ? In other words, is it true that for every $f \in L^{1}(m)$,

$$
\lim _{\eta} I_{m_{\eta}}(f)=I_{m}(f) ?
$$

We will show in what follows that in general the answer is negative, although it is true for $L^{p}[0,1], p \geq 1$.

Example 3.9. Let us check again our canonical example: the identity map in $L^{1}[0,1]$ considered as an integration map for the vector measure $m(A):=\chi_{A}, A \in \Sigma$. Fix a function $f \in L^{1}(m)$. Consider the net $\mathbb{P}$ and fix $\varepsilon>0$. Then there is a simple function $f_{\varepsilon}=\sum_{i=1}^{n} \lambda_{i} \chi_{B_{i}}$ such that $\left\|f-f_{\varepsilon}\right\|_{L^{1}(m)} \leq \varepsilon$. It is assumed, by putting $\lambda_{n}=0$ if necessary, that $\mathcal{P}_{\eta_{0}}=\left\{B_{1}, \ldots, B_{n}\right\}$ is a partition. Note that $I_{m_{\eta}}\left(f_{\varepsilon}\right)=I_{m_{\eta_{0}}}\left(f_{\varepsilon}\right)=f_{\varepsilon}$ for every $\eta \geq \eta_{0}$. Then

$$
\begin{aligned}
\left\|I_{m}(f)-I_{m_{\eta}}(f)\right\|_{L^{1}[0,1]} & \leq\left\|I_{m}(f)-I_{m_{\eta}}\left(f_{\varepsilon}\right)\right\|_{L^{1}[0,1]}+\left\|I_{m_{\eta}}\left(f_{\varepsilon}\right)-I_{m_{\eta}}(f)\right\|_{L^{1}[0,1]} \\
& \leq \varepsilon+\left\|f_{\varepsilon}-\sum_{i=1}^{n} \frac{\int_{B_{i}} f d \mu}{\mu\left(B_{i}\right)} \chi_{B_{i}}\right\|_{L^{1}[0,1]} \\
& =\varepsilon+\left\|\sum_{i=1}^{n}\left(\lambda_{i}-\frac{\int_{B_{i}} f d \mu}{\mu\left(B_{i}\right)}\right) \chi_{B_{i}}\right\|_{L^{1}[0,1]} \\
& =\varepsilon+\int \sum_{i=1}^{n} \mid \lambda_{i}-\frac{\int_{B_{i}} f d \mu}{\mu\left(B_{i}\right)} \chi_{B_{i}} d \mu \\
& =\varepsilon+\sum_{i=1}^{n}\left|\lambda_{i} \mu\left(B_{i}\right)-\int_{B_{i}} f d \mu\right| \\
& =\varepsilon+\sum_{i=1}^{n}\left|\int_{B_{i}} f_{\varepsilon} d \mu-\int_{B_{i}} f d \mu\right| \\
& \leq \varepsilon+\sum_{i=1}^{n} \int_{B_{i}}\left|f_{\varepsilon}-f\right| d \mu \\
& =\varepsilon+\left\|f_{\varepsilon}-f\right\|_{L^{1}[0,1]} \leq 2 \varepsilon .
\end{aligned}
$$

Therefore, we have that $\lim _{\eta} I_{m_{\eta}}=I_{m}$ pointwise. Moreover, notice that for each $A \in \Sigma$, if we replace $f$ and $f_{\varepsilon}$ by $f h_{A}$ and $f_{\varepsilon} h_{A}$ respectively in the computations above, we can also prove that for each $f \in L^{1}(m)$,

$$
\limsup _{\eta \in \Sigma}\left\|\int f h_{A} d m-\int f h_{A} d m_{\eta}\right\|=0 .
$$

Thus, by Theorem 3.4(i) we obtain $\lim _{\eta}\|f\|_{L^{1}\left(m_{\eta}\right)}=\|f\|_{L^{1}(m)}$ for each $f \in L^{1}(m)$.

In general, the convergence of martingales is not assured in Banach function spaces. It is well known that in the case of the spaces $L^{p}[0,1], 1<p<\infty$, this is true, as a consequence of Doob's martingale inequality; actually, this fact can be extended to the case of Bochner spaces $L^{p}(\mu, X)$ over probability nonatomic measures $\mu$ (the reader can find a proof in Theorem 1.5 and Remark 1.7 in [15]). However, the arguments that support these results cannot be transferred to the whole class of Banach function spaces. Consider an order continuous Banach function space $X(\mu)$ over a probability non-atomic measure $\mu$, and define the same vector measure $\Sigma \ni A \mapsto \chi_{A} \in X(\mu)$ that has been considered in the example above. 
Note that in this case $L^{1}(m)=X(\mu)$ isometrically and $I_{m}=\mathrm{Id}$, the identity map in $X(\mu)$. In [10, it is studied to what extent the convergence of martingales in Banach function spaces resembles the case of $L^{p}[0,1]$, and several positive results are shown for relevant spaces like $L \log L$. However, a counterexample is also given for the general fact. It must be noted that in this paper, the definition of Banach function space includes the requirement of having the Fatou property, so the class of Banach function spaces considered there is smaller than the one we are considering.

Under this requirement, Theorem 1 in [10] establishes that for an order continuous (and Fatou) Banach function space $X(\mu)$, a uniformly integrable martingale defined by a sequence of continuous conditional expectation operators $E_{n}: X(\mu) \rightarrow$ $X(\mu)$ when applied to a function $f \in X(\mu)$ is convergent to $f$ in the norm of $X(\mu)$ for each $f$, if and only if the sequence $\left(\left\|E_{n}\right\|\right)_{n}$ is uniformly bounded. However, it is also proved that this requirement is not always satisfied. Thus, every sequence of conditional expectation operators is bounded in $L^{p}$, but in a general function space this is not true. Moreover, Theorem 2 in [10] shows that for rearrangement invariant order continuous and Fatou Banach function spaces the convergence of uniformly integrable martingales is guaranteed. Again, there are spaces $L^{1}(m)$ that are not rearrangement invariant, so the result does not apply in our setting.

\section{The Daugavet equation for integration maps}

In this section we analyse the negative results regarding unconditional pointwise approximation of integration maps. We will show that, even in the simplest examples, the integration map of a vector measure cannot be approximated pointwise as an unconditional series of finite rank operators, or even of weakly compact operators. The main conclusion of this section is the following. There are vector measures with associated integration maps of absolutely different nature representing the same space: they may satisfy that $\|f\|_{L^{1}(m)}=\lim _{\eta}\|f\|_{L^{1}\left(m_{\eta}\right)}$ for all $f \in L^{1}(m)$, but $I_{m}$ cannot be represented as an unconditionally pointwise convergent series of weakly compact operators. The Daugavet property is our main source of examples and counterexamples, together with the following useful result that can be found in [4, Th. 2.9].

Theorem A. Let $G \in L(X, Y)$. Suppose that the inequality

$$
\|G+T\| \geq C+\|T\|
$$

with $C>0$ holds for every operator $T$ from a subspace $M \subseteq L(X, Y)$ of operators. Let $\hat{T}=\sum_{n \in \Gamma} T_{n}$ be a (maybe uncountable) pointwise unconditionally convergent series of operators $T_{n} \in M$. Then $\|G-\hat{T}\| \geq C$.

Recall that if a Banach space $X$ has the Daugavet property, then we have that for every weakly compact operator, $\|\operatorname{Id}+T\|=1+\|T\|$, so the result above applies; this result can be found in [9, Lemma 2.6] for this specific case.

Let $(\Omega, \Sigma)$ be a measurable space and $\mu$ a positive measure without atoms. The main examples of Banach function spaces that have the Daugavet property are $L^{1}(\mu)$ and $L^{\infty}(\mu)$. Another example is $C(K)$, if $K$ is a compact Hausdorff topological space without isolated points. In this section we study when the integration operator $I_{m}$ satisfies the Daugavet equation for a suitable $L^{1}(m)$-valued vector measure or in a more general sense, when $\left\|I_{m}+I_{n}\right\|=\left\|I_{m}\right\|+\left\|I_{n}\right\|$ for $m$ and $n$ being vector measures $m, n: \Sigma \rightarrow X$ such that $L^{1}(m)=L^{1}(n)$. 
Let us start with the main negative example. Let $m$ be a non-atomic vector measure. It is well known that $I_{m}$ is compact if and only if $m$ has finite variation and admits a Radon-Nikodým derivative, and in this case $L^{1}(m)=L^{1}(|m|)$ (see [14. Ch. 3]). For instance, if $\mu$ is a Rybakov measure for such a vector measure $m$ satisfying $L^{1}(m)=L^{1}(\mu)$, then the integration map $I_{\mu}: L^{1}(m) \rightarrow \mathbb{R}$ given by $f \mapsto \int f d \mu$ produces the same space of integrable functions. If we fix a norm one function $g \in L^{1}(\mu)$, we can consider this integration map as having values in $L^{1}(\mu)$ by defining it as $f \mapsto \int f d \mu \otimes g$.

However, the operator associated to the vector measure $m_{0}(A):=\chi_{A} \in L^{1}(\mu)$, that is the identity map $I_{m_{0}}=\mathrm{Id}: L^{1}(m) \rightarrow L^{1}(\mu)$, gives the same space of integrable functions $L^{1}\left(m_{0}\right)=L^{1}(m)=L^{1}(\mu)$. In this case, it is clear that the integration map $I_{m_{0}}=$ Id cannot be approximated in the operator norm by a sequence of compact operators.

Theorem A above, together with the Daugavet property of $L^{1}[0,1]$, gives that the integration map $I_{m_{0}}: L^{1}[0,1] \rightarrow L^{1}[0,1]$ cannot be approximated by any pointwise unconditional sum of weakly compact operators. However, if $m_{1}(A):=\mu(A) \otimes \chi_{[0,1]}$ - which has an associated rank 1 integration map -, we have that $L^{1}\left(m_{0}\right)=$ $L^{1}\left(m_{1}\right)=L^{1}[0,1]$ and for each function $f \in L^{1}[0,1],\|f\|_{L^{1}\left(m_{0}\right)}=\|f\|_{L^{1}\left(m_{1}\right)}$. The results provided in Section 3.4 show that, although we can approximate $I_{m_{0}}$ pointwise by a net of finite rank operators, the corresponding series that approximates a function cannot be unconditionally convergent in general.

Let us show another example in this direction.

Example 4.1. The previous example gives some ideas regarding spaces $L^{1}(m)$ of $L^{1}(\mu)$-valued measures. Let $(\Omega, \Sigma, \mu)$ be a non-atomic finite measure space. For a continuous linear operator $T: L^{1}(\mu) \rightarrow L^{1}(\mu)$ consider the vector measure defined as $m_{T}(A):=T\left(\chi_{A}\right)$ for each measurable set $A \in \Sigma$.

Let $T: L^{1}(\mu) \rightarrow L^{1}(\mu)$ be an isomorphism and let $R: L^{1}(\mu) \rightarrow L^{1}(\mu)$ be a Radon-Nikodým operator (for example, a weakly compact operator). Consider the vector measures $m_{T}$ and $m_{R \circ T}$. Then the corresponding integration operators $I_{m_{T}}$ and $I_{m_{R \circ T}}$ satisfy

$$
\left\|I_{m_{T}}+I_{m_{R \circ T}}\right\|=\left\|I_{m_{T}}\right\|+\left\|I_{m_{R \circ T}}\right\|
$$

Therefore, for each $\varepsilon>0$ there is a function $f \in L^{1}\left(m_{T}\right)$ such that

$$
\left\|\int f d m_{T}-\int f d m_{R \circ T}\right\| \geq\left\|I_{m_{T}}\right\|+\left\|I_{m_{R \circ T}}\right\|-\varepsilon .
$$

In other words, again by Theorem A we cannot approximate pointwise unconditionally the integration map associated to $T$ by means of integration maps associated to vector measures constructed using operators like $R \circ T$, where $R$ is a RadonNikodým operator.

As in the previous section, in what follows we will describe the Daugavet equation among integration operators in terms of the Radon-Nikodým derivative operators of their scalarizations. As usual, the restriction of an operator $T: E \rightarrow X$ to a subset $Y$ of $E$ is denoted by $\left.T\right|_{Y}$.

Lemma 4.2. Let $X_{0}, X_{1}$ be Banach spaces and consider two vector measures $m$ : $\Sigma \rightarrow X_{0}$ and $m_{1}: \Sigma \rightarrow X_{1}$ that are equivalent to a scalar measure $\mu$. Suppose that $Z=Z(\mu)$ is a Banach function space such that $Z(\mu) \subseteq L^{1}(m) \cap L^{1}\left(m_{1}\right)$ and 
$X_{0}+X_{1} \subseteq X$ with continuous inclusions. Then for every scalar $\lambda$,

$$
\left\|\left.I_{m}\right|_{Z}+\left.\lambda I_{m_{1}}\right|_{Z}\right\|_{L(Z(\mu), X)}=\sup _{x^{*} \in B_{X^{*}}}\left\|\varphi_{x^{*}}^{m}+\lambda \varphi_{x^{*}}^{m_{1}}\right\|_{Z(\mu)^{*}}
$$

Proof. Note that by the inclusion requirement $Z(\mu) \subseteq L^{1}(m) \cap L^{1}\left(m_{1}\right)$, for all $f \in Z(\mu)$ we have that $\int f d m$ and $\int f d m_{1}$ make sense. The following direct computation gives the result.

$$
\begin{aligned}
\left\|\left.I_{m}\right|_{Z}+\lambda I_{m_{1}} \mid Z\right\|_{L(Z, X)} & =\sup _{f \in B_{Z}}\left\|\left.I_{m}\right|_{Z}(f)+\left.\lambda I_{m_{1}}\right|_{Z}(f)\right\|_{X} \\
& =\sup _{f \in B_{Z}}\left\|\int f d m+\lambda \int f d m_{1}\right\|_{X} \\
& =\sup _{f \in B_{Z}} \sup \left\{\left\langle\int f d m, x^{*}\right\rangle+\left\langle\int \lambda f d m_{1}, x^{*}\right\rangle: x^{*} \in B_{X^{*}}\right\} \\
& =\sup _{f \in B_{Z}, x^{*} \in B_{X^{*}}}\left\{\int f d\left\langle m, x^{*}\right\rangle+\lambda \int f d\left\langle m_{1}, x^{*}\right\rangle\right\} .
\end{aligned}
$$

Let $\varphi_{x^{*}}^{m}=\frac{d\left\langle m, x^{*}\right\rangle}{d \mu}$ and $\varphi_{x^{*}}^{m_{1}}=\frac{d\left\langle m_{1}, x^{*}\right\rangle}{d \mu}$ be the Radon-Nikodým derivatives with respect to the fixed measure $\mu$; note that they belong to $Z^{*}$. Then

$$
\begin{aligned}
\sup _{f \in B_{Z}, x^{*} \in B_{X^{*}}}\left\{\int f d\left\langle m, x^{*}\right\rangle\right. & \left.+\lambda \int f d\left\langle m, x^{*}\right\rangle\right\} \\
& =\sup _{f \in B_{Z}, x^{*} \in B_{X^{*}}}\left\{\int f \varphi_{x^{*}}^{m} d \mu+\int \lambda f \varphi_{x^{*}}^{m_{1}} d \mu\right\} \\
& =\sup _{f \in B_{Z}, x^{*} \in B_{X^{*}}}\left\{\int f\left(\varphi_{x^{*}}^{m}+\lambda \varphi_{x^{*}}^{m_{1}}\right) d \mu\right\} \\
& =\sup _{x^{*} \in B_{X^{*}}}\left\|\varphi_{x^{*}}^{m}+\lambda \varphi_{x^{*}}^{m_{1}}\right\|_{Z^{*}} .
\end{aligned}
$$

Let us show some direct applications. Let $X(\mu)$ be an order continuous Banach function space over a finite measure space $(\Omega, \Sigma, \mu)$. We will say that a vector measure $m: \Sigma \rightarrow X$ represents $X(\mu)$ if $L^{1}(m)=X(\mu)$ isometrically and in the order. We consider first the case when a space $X(\mu)$ is represented by a vector measure $m$ having values in the same space $X(\mu)$. Thus, let us show a particular case that will be relevant later on in the paper. Suppose that $m$ and $m_{1}$ are vector measures having values in the order continuous Banach function space $X(\mu)$ such that $L^{1}(m)=L^{1}\left(m_{1}\right)=X(\mu)$ (isometrically), and in such a way that $I_{m_{1}}=$ Id : $X(\mu) \rightarrow X(\mu)$, the identity map; this happens if $m_{1}$ is the vector measure $\Sigma \ni A \mapsto m_{1}(A):=\chi_{A} \in X(\mu)$, and in this case $\operatorname{Id}(f)=\int f d m_{1}=f$. Then,

$$
\left\|\mathrm{Id}+I_{m}\right\|=\sup _{h \in B_{(X(\mu))^{\prime}}}\left\|h+\varphi_{h}^{m}\right\|,
$$

and consequently:

Corollary 4.3. For each vector measure $m: \Sigma \rightarrow X(\mu)$ representing $X(\mu)$, the Daugavet equation $\left\|\mathrm{Id}+I_{m}\right\|=2$ holds if and only if

$$
\sup _{h \in B_{(X(\mu))^{\prime}}}\left\|h+\frac{d\langle m, h\rangle}{d \mu}\right\|=2
$$

holds. 
Example 4.4. Consider again our canonical example. Take the $L^{1}[0,1]$-valued vector measures defined as $m_{1}(A):=\chi_{A}$ and $m(A):=\mu(A) \cdot \chi_{[0,1]}$ for each Lebesgue measurable set $A$. Both of them give $L^{1}[0,1]$ as space of integrable functions isometrically; for them, we have that

$$
\sup _{h \in B_{L} \infty[0,1]}\left\|h+\frac{d\langle m, h\rangle}{d \mu}\right\|=2 .
$$

Taking into account that the Radon-Nikodým derivative of the measure $A \mapsto$ $\mu(A) \int \chi_{[0,1]} h d \mu=\langle m, h\rangle(A)$ is

$$
\frac{d\langle m, h\rangle}{d \mu}=\chi_{[0,1]} \int_{[0,1]} h d \mu,
$$

we obtain that in this case, this is equivalent to

$$
\sup _{h \in B_{L} \infty[0,1]}\left\|h+\chi_{[0,1]} \int_{[0,1]} h d \mu\right\|=2
$$

in fact, the same result is true when we replace the function $\chi_{[0,1]}$ by any other norm one function of $L^{1}[0,1]$, since all of them define rank 1 operators.

There are other cases of Banach lattices of integrable functions that also have the Daugavet property and are not $L^{1}$-spaces. Let us explain some examples. It is well known that, if $\mu$ is a nonatomic probability measure and $E$ is a Banach space, the space $L^{1}(\mu, E)$ of Bochner integrable functions has the Daugavet property (see 9. p. 858, Example]). Thus, in the case that $E$ is also a Banach lattice, it is known that $L^{1}(\mu, E)$ is a Banach lattice too that has the Daugavet property. Another interesting example of a Banach space having the Daugavet property is the Bochner space $L^{\infty}(\mu, E)$ : it has the Daugavet property is $E$ has it or $\mu$ is nonatomic (see [13, Th. 5]) it is also a Banach lattice if $E$ is so. However, since all the spaces $L^{1}(m)$ for a vector measure $m$ are order continuous Banach function spaces, the application of the previously mentioned space as example of our class is restricted to finite $\ell^{\infty}$-sums of Banach function spaces having the Daugavet property (this case was already considered in [17). Anyway, $c_{0}$-sums of Banach function spaces with the Daugavet property have also this property, as can be deduced from Proposition 2.16 in [9]: take for example a disjoint countable measurable partition $\left\{A_{i}: i \in \mathbb{N}\right\}$ of $[0,1]$; the $c_{0}$-sum of $L^{1}\left(\left.\mu\right|_{A_{i}}\right)$ has the Daugavet property. This space satisfies the order continuity requirement, and so it can be represented as an $L^{1}(m)$ of a vector measure $m$.

However, it must be said that the class of Banach function spaces having the Daugavet property is rather small. The results in [1, 2] and [8] show this. Thus, Theorem 3.6 in [2] states that for a rearrangement invariant Banach function space $X(\mu)$ over a finite measure $\mu$ with the weak Fatou property, the Daugavet property implies that $X(\mu)$ coincides either with $L^{\infty}(\mu)$ or with $L^{1}(\mu)$ isometrically. Since all the spaces $L^{1}(m)$ of a vector measure $m$ are order continuous, our class is restricted to the case of $L^{1}$-spaces of finite measures. Moreover, if $L^{1}(m)$ is an Orlicz space with the Luxemburg norm, for $m$ being non-atomic, and has the Daugavet property, then it must be isometric to $L^{1}$ [2, Cor. 4.3].

Therefore we obtain the following result. 
Proposition 4.5. Let $X(\mu)$ be an order continuous Banach function space with the Daugavet property (in particular, if $X(\mu)=L^{1}(\mu)$ for a non-atomic measure $\mu)$. The following assertions hold.

(1) Let $m$ be an $X(\mu)$-valued countably additive vector measure representing $X(\mu)$ such that $I_{m}$ is a Radon-Nikodým operator. Then

$$
\left\|\mathrm{Id}+I_{m}\right\|=\sup _{h \in B_{(X(\mu))^{\prime}}}\left\|h+\lambda \varphi_{h}^{m}\right\|_{(X(\mu))^{\prime}}=2 .
$$

(2) There is a vector measure $m$ representing $X(\mu)$ such that its integration map $I_{m}$ is not a pointwise unconditional sum (maybe uncountable) of RadonNikodým operators. In fact, the same result holds if $X(\mu)$ is isomorphic to a space with the Daugavet property.

(3) Let $\mathcal{M}$ be the normed space of all linear combinations of finite rank RadonNikodým derivative operators associated to vector measures representing $X(\mu)$. Then the integration map associated to the vector measure $\Sigma \ni A \mapsto$ $\chi_{A} \in X(\mu)$ representing $X(\mu)$ - the identity map - cannot be approximated as a (maybe uncountable) pointwise unconditionally convergent series of operators in $\mathcal{M}$.

Proof. The first statement is a direct consequence of the Daugavet property of $X(\mu)$. The second one can be proved as a direct application of Theorem 2.9 in [4, taking into account that again by the Daugavet property of $X(\mu)$, RadonNikodým operators satisfy the Daugavet equation. The third statement is the result of considering in particular those operators that are finite sums of finite rank integration maps representing $X(\mu)$.

Remark 4.6. A similar version of the result above can be obtained if we replace the operator Id by any integration operator $I_{m}$ that is a Daugavet center, without the assumption that $X(\mu)$ has the Daugavet property. The reader can find information about this notion in 3 and 4 . In fact, using Remark 2.10 in 4, more can be said. If a Banach function space $X(\mu)$ can be represented by an integration map maybe defined in a different Banach space $F$ - that is a Daugavet center, then $X(\mu)$ cannot have an unconditional basis (countable or uncountable) or be represented as unconditional sum of reflexive spaces. The reason is that then $I_{m}$ cannot be written as a pointwise unconditionally convergent series of weakly compact operators; in particular, of course $I_{m}$ cannot be a weakly compact operator.

The structure of the spaces $L^{1}(m)$ with $I_{m}$ being a Daugavet center is, as a consequence of the previous results and comments, close to being isomorphic to spaces $L^{1}$ of scalar measures. In fact, in [4] it is proved that every Daugavet center fixes a copy of $\ell^{1}$, and so $L^{1}(m)$ cannot be a reflexive space.

\section{REFERENCES}

[1] M.D. Acosta, A. Kamińska, and M. Mastyło, The Daugavet property and weak neighborhoods in Banach lattices, J. Convex Anal., 19(3) (2012), 875-912.

[2] M.D. Acosta, A. Kamińska, and M. Mastyło, The Daugavet property in rearrangement invariant spaces, Trans. Amer. Math. Soc. 367(6) (2015), 4061-4078.

[3] T.V. Bosenko, Daugavet centers and direct sums of Banach spaces, Cent. Eur. J. Math. 8(2) (2010), 346-356.

[4] T.V. Bosenko and V. Kadets, Daugavet centers, J. Math. Phys. Anal. Geom. 6(1) (2010), $3-20$. 
[5] S. Brach, E.A. Sánchez Pérez, and D. Werner, The Daugavet equation for bounded vector valued functions, to appear in Rocky Mountain J. Math.

[6] J. Diestel and J.J. Uhl, Jr., Vector Measures, Mathematical Surveys, 15, Amer. Math. Soc. Providence, R.I., 1977.

[7] A. Grothendieck, Produits tensoriels topologiques et espaces nucléaires, Mem. Amer. Math. Soc. 16 (1955).

[8] V. Kadets, M. Martín, J. Merí, and D. Werner, Lushness, numerical index 1 and the Daugavet property in rearrangement invariant spaces, Can. J. Math. 65(2) (2013), 331-348.

[9] V.M. Kadets, R.V. Shvidkoy, G.G. Sirotkin, and D. Werner, Banach spaces with the Daugavet property, Trans. Amer. Math. Soc. 352(2) (2000), 855-873.

[10] M. Kikuchi, A note on the convergence of martingales in Banach function spaces, Analysis Mathematica, 25 (1999), 265-276.

[11] Å. Lima, O. Nygaard, and E. Oja, Isometric factorization of weakly compact operators and the approximation property, Israel J. Math. 119 (2000), 325-348.

[12] Å. Lima and E. Oja, Ideals of operators, approximability in the strong operator topology, and the approximation property, Michigan Math. J. 52(2) (2004), 253-265.

[13] M. Martín and A.R. Villena, Numerical index and the Daugavet property for $L_{\infty}(\mu, X)$, Proc. Edinb. Math. Soc., II. Ser. 46(2) (2003), 415-420.

[14] S. Okada, W. Ricker, and E.A. Sánchez Pérez, Optimal Domains and Integral Extensions of Operators Acting in Function Spaces, Operator Theory. Advances and Applications, Vol. 180, Birkhäuser, Basel, 2008.

[15] G. Pisier, Martingales in Banach Spaces, Cambridge University Press, Cambridge, 2016.

[16] D. Werner, Recent progress on the Daugavet property, Irish Math. Soc. Bulletin 46 (2001), $77-97$.

[17] P. Wojtaszczyk, Some remarks on the Daugavet equation, Proc. Amer. Math. Soc. 115(4) (1992), 1047-1052.

E. Jiménez Fernández, Departamento de Economía, Universitat Jaume I, Campus del Riu Sec, s/n, 12071 Castelló de la Plana, Spain

E-mail address: jimeneze@uji.es

E.A. Sánchez Pérez, Instituto Universitario de Matemática Pura y Aplicada, Universitat Politècnica de València, Camino de Vera s/n, 46022 Valencia, Spain

E-mail address: easancpe@mat.upv.es

D. Werner, Fachbereich Mathematik und Informatik, Freie Universitat Berlin, Arnimallee 6, 14195 Berlin, Germany

E-mail address: werner@math.fu-berlin.de 